\title{
Capecitabine plus oxaliplatin compared with 5-fluorouracil plus oxaliplatin in metastatic colorectal cancer: Meta-analysis of randomized controlled trials
}

\author{
CHENGYAO ZHANG $^{1 *}$, JIAWU WANG $^{2 *}$, HAITAO GU $^{1 *}$, DAIHUA ZHU ${ }^{1}$, \\ YANG $\mathrm{LI}^{1}$, PENG ZHU ${ }^{1}$, YAXU WANG ${ }^{1}$ and JIJIAN WANG ${ }^{1}$ \\ ${ }^{1}$ Department of General Surgery, The Second Affiliated Hospital of Chongqing Medical University, \\ Chongqing 400010; ${ }^{2}$ Department of Urology, Chongqing Steel Group General Hospital of Chongqing, \\ Chongqing 400080, P.R. China
}

Received November 16, 2011; Accepted January 4, 2012

DOI: $10.3892 / \mathrm{ol} .2012 .567$

\begin{abstract}
The aim of this study was to evaluate the curative effects and safety of capecitabine plus oxaliplatin compared with 5-fluorouracil (5-FU) plus oxaliplatin in patients with metastatic colorectal cancer (MCRC). We searched the Cochrane Central register of Controlled Trials (CENTRAL), PubMed, Ovid, ScienceDirect, EBSCO, EMBASE and conference proceedings for eligible trials. A meta-analysis was performed using Review Manager 5.0. A total of 3,603 cancer patients from 7 trials were analyzed, and the baseline patient characteristics were comparable in all studies. Curative effect outcomes including complete response (CR) $(\mathrm{OR}=0.78$; $95 \%$ CI 0.47-1.31; $\mathrm{p}=0.35)$, partial response $(\mathrm{PR})(\mathrm{OR}=0.81 ; 95 \%$ CI $0.65-1.00$; $\mathrm{p}=0.05)$ and the overall response rate $(\mathrm{ORR})(\mathrm{OR}=0.85 ; 95 \%$ CI 0.71-1.02; $\mathrm{p}=0.08$ ) showed similar curative effects between the capecitabine plus oxaliplatin group and the 5-FU plus oxaliplatin group. Moreover, the median overall survival (OS) and progression-free survival (PFS) had no statistically significant differences. Regarding safety, hand-foot syndrome was more frequently observed in the capecitabine plus oxaliplatin group $(\mathrm{OR}=2.71 ; 95 \%$ CI 2.04-3.61; $\mathrm{p}<0.00001)$, while stomatitis and neutropenia were reversed. Other toxic effects had no statistically significant differences between the two groups. Our results showed that capecitabine plus oxaliplatin had similar curative effects to 5-FU plus oxaliplatin, however, it was safer in patients with MCRC.
\end{abstract}

Correspondence to: Dr Jijian Wang, Department of General Surgery, The Second Affiliated Hospital of Chongqing Medical University, Chongqing 400010, P.R. China

E-mail: wangjijian1963@yahoo.cn

${ }^{*}$ Contributed equally

Key words: capecitabine, oxaliplatin, metastatic colorectal cancer, meta-analysis

\section{Introduction}

Colorectal cancer (CRC) is one of the most common causes of digestive system cancer-related mortality, and is the fourth main cause of cancer-related mortality worldwide (1). In Western countries, metastatic colorectal cancer (MCRC) is the second most frequently diagnosed form of malignant tumor. In recent years, the incidence of CRC has increased markedly, and $50 \%$ of patients eventually succumb to the disease due to metastatic spread of the cancer. Surgery is the standard treatment for resectable CRC if the disease has not spread prior to surgery. However, chemotherapy was required for MCRC patients to prolong the median survival. Numerous new drugs and chemotherapy regimens have been used in CRC therapy, which have improved the survival rate and reduced the adverse drug reactions.

Combinations of 5-fluorouracil/folinic acid (5-FU/FA) and oxaliplatin (FOLFOX regimens) are established standard regimens for the first-line treatment of MCRC (2). Capecitabine (Xeloda; Hoffmann-La Roche Inc., Nutley, NJ, USA) is an oral fluoropyrimidine that has similar efficacy to bolus 5-FU/ FA as a monotherapy for the first-line treatment of MCRC, but has a high target-specific killing effect on tumor cells of the human body. Previous studies confirmed that capecitabine plus oxaliplatin is non-inferior to $5-\mathrm{FU} / \mathrm{LV}$ for the treatment of CRC (3). In this study, a large of database of patients with MCRC who were eligible for clinical trials was collected to assess the curative effects and safety of capecitabine plus oxaliplatin compared with 5-FU plus oxaliplatin in patients with MCRC.

\section{Materials and methods}

Search strategy. We searched the Cochrane Central register of Controlled Trials (CENTRAL), PubMed, Ovid, ScienceDirect, EBSCO, EMBASE and conference proceedings for eligible trials between January 2000 and April 2011. Searches were conducted using the following MESH terms: 'capecitabine', 'oxaliplatin', 'colorectal neoplasms', 'FOLFOX' and 'XELOX', as well as the text words: metastatic colorectal 
cancer, colorectal carcinoma and chemotherapy. Searches were limited to literature in English.

Inclusion criteria. Inclusion criteria for retrieved studies were: i) Studies were randomized controlled trials (RCTs), and published in English; ii) patients had histologically confirmed CRC; iii) in intervention studies, the experimental group patients underwent a capecitabine plus oxaliplatin regimen, consisting of a 2 -h intravenous infusion of oxaliplatin $130 \mathrm{mg} / \mathrm{m}^{2}$ on day 1 plus oral capecitabine $1,000 \mathrm{mg} / \mathrm{m}^{2}$ twice daily on days 1 to 14 every 3 weeks; in the control group patients underwent a 5 -FU plus oxaliplatin regimen. The chemotherapy dose and cycle of 5-FU and oxaliplatin were not limited as there were a number of regimens applied in various clinics, for example, FOLFOX4, FOLFOX6, FUFOX and FUOX (4). Original literature outcomes included overall response rate (ORR), progression-free survival (PFS), median overall survival (OS) and toxic effects evaluated according to National Cancer Institute (NCI) common terminology criteria for adverse events (CTCAE).

Exclusion criteria. Exclusion criteria for retrieved studies were: i) A lack of basic data necessary for our research or that the basic data were incomplete; ii) we chose the most recent literature if there was repetition of the same or similar reports.

Data collection. The two reviewers extracted data on: i) Basic patient characteristics, such as age, gender, the primary tumor sites, tumor status, number of metastatic sites and the Eastern Cooperative Oncology Group scale performance status (ECOG PS); ii) description of interventions, outcomes (CR, PR, ORR, OS, PFS and the adverse reaction of patients according to CTCAE) and lost to follow-up; iii) study design: Concealment of treatment allocation and blinding.

Quality assessment. The study quality was judged by the same two reviewers according to a modified Jadad score (4). Assessment scores were as follows: i) Randomization method: 2 points for appropriate, 1 point for not clear, 0 point for inappropriate; ii) blinding of outcomes: 2 points for appropriate, 1 point for not clear, 0 point for inappropriate; iii) description of follow-up situation if the patients were lost to follow-up, exit from or breach of the treatment regimen: 1 point for intentionto-treat analysis, 0 point for no description. Total scores were 1 to 5 points: studies with 1-2 points were classified as low quality studies, and those with 3-5 points were classified as high quality studies. Any disagreement in the extracted data was resolved by a third reviewer.

Statistical analysis. Statistical analyses were performed using the Review Manager 5.0 freeware package. The heterogeneity of trials was estimated by use of the Chi-square test. A random effects model was preferred for heterogeneous data and a Mantel-Haenszel fixed effects model for homogeneous data. The odds ratio (OR) and 95\% confidence interval (95\% CI) were used as summary statistics for categorical variables. Analyses were according to the intention-to-treat principle. Results were presented in all figures as conventional meta-analysis forest plots. A two-sided p-value of $<0.05$ was judged to indicate a statistically significant difference for all analyses.

\section{Results}

Search results and study selection. We searched more than 170 references in CENTRAL, PubMed, Ovid, ScienceDirect, EBSCO, EMBASE and conference proceedings. Following an initial screening of the title and abstract, 33 studies were thought to meet the inclusion criteria. Following further screening of full texts, we excluded 28 articles due to a lack of basic data or disagreement of outcome measures. Finally, seven studies published between 2000 and 2011, including 3,603 cancer patients, comprising 1,702 in the capecitabine plus oxaliplatin group (experimental group) and 1,901 in the 5-FU plus oxaliplatin group (control group), were included in the meta-analysis. The complete articles were retrieved. Trial characteristics are shown in Table I.

Seven RCTs were observed for PFS and OS (3,603 patients, intention-to-treat analyses) for the primary endpoints. With regard to the secondary endpoints, four trials were evaluated for CR and PR (1444 patients) and five trials for ORR (2071 patients).

Quality assessment. The Jadad scale assessed all RCTs and this information was listed in Table II. None of the trials had any description of concealment of treatment allocation and blinding methods, but they had described the randomization method and follow-up situation appropriately. Analyses were according to the intention-to-treat principle.

Curative effects analysis. Among the seven included trials, four studies reported on CR and PR. A meta-analysis was performed on the four studies. The heterogeneity test did not reveal any significant departure from the Chi-square test (CR: $\mathrm{p}=0.06, \mathrm{I}^{2}=59 \%$; PR: $\mathrm{p}=0.84, \mathrm{I}^{2}=0 \%$ ), thus, OR calculations were performed according to the fixed effects model. The results showed that there was no statistically significant difference between the two regimens on $\mathrm{CR}$ and $\mathrm{PR}(\mathrm{OR}=0.78,95 \%$ CI $0.47-1.31, \mathrm{p}=0.35$, Fig. $1 ; \mathrm{OR}=0.81,95 \%$ CI $0.65-1.00$, $\mathrm{p}=0.05$; Fig. 2).

ORR was selected as the outcome measure in the five included articles. No significant heterogeneity was present in ORR ( $p=0.34, \mathrm{I}^{2}=11 \%$ ). Using a fixed effects model, the result showed that the differences between the capecitabine plus oxaliplatin group and the 5-FU plus oxaliplatin group were not statistically significant $(\mathrm{OR}=0.85,95 \%$ CI $0.70-1.02, \mathrm{p}=0.08$; Fig. 3). Comprehensive analysis of these results showed the similar curative effects of capecitabine plus oxaliplatin compared with 5-FU plus oxaliplatin for MCRC.

PFS and OS analysis. We did not obtain information from each individual patient from every study. Furthermore, PFS and OS belong to the skewed distribution that cannot be studied by meta-analysis. Therefore, we only performed a descriptive statistical analysis of the data. All seven studies reported PFS and median OS (Table III).

Adverse reaction analysis. The most commonly reported chemotherapy-related adverse events were nausea, vomiting, diarrhea, fever, thrombocytopenia, neutropenia, stomatitis, hand-foot syndrome and peripheral neuropathy. There were five studies reporting the toxic effects of hand-food syndrome. 


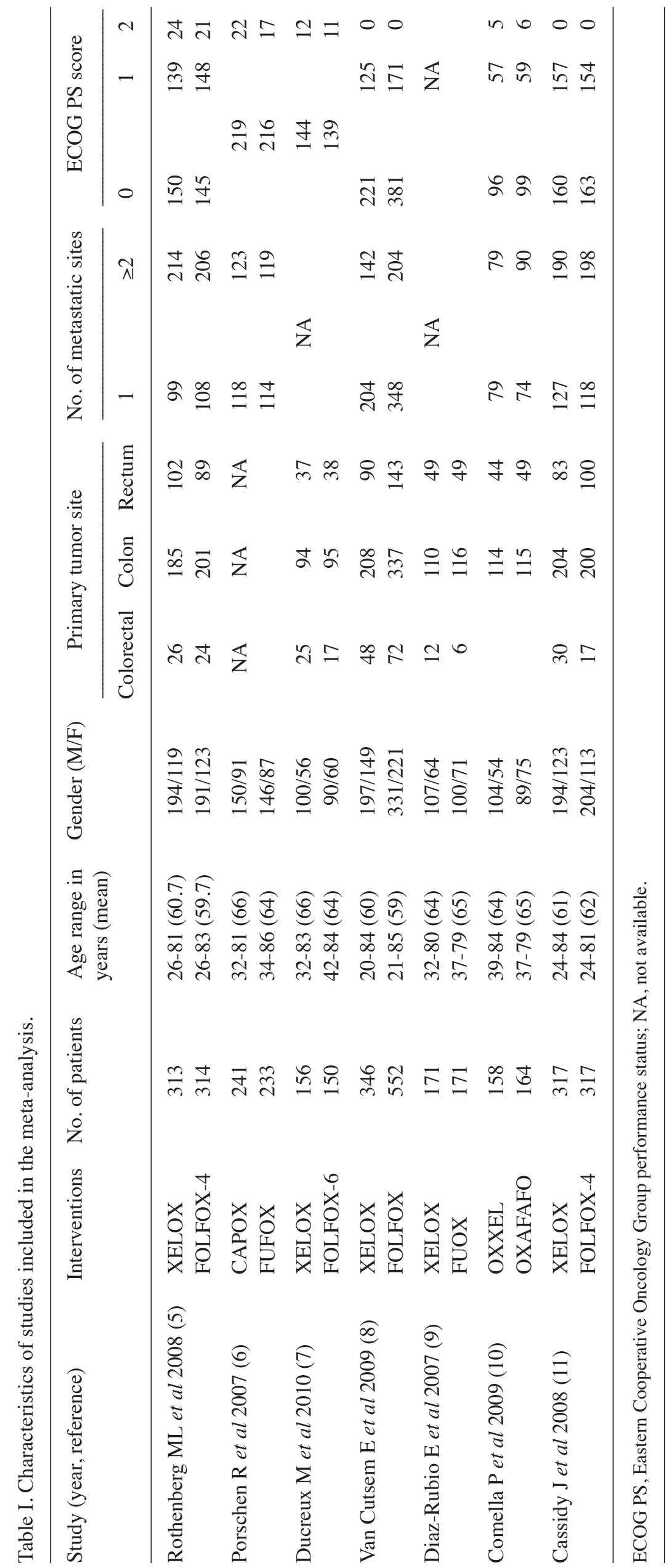


Table II. Quality assessment of studies included in the meta-analysis.

\begin{tabular}{lccccc}
\hline Study (year, reference) & Randomization & Blind & Exit/lose & ITT & Jadad score \\
\hline Rothenberg ML et al 2008 (5) & Yes & NA & Yes & Yes & 4 \\
Porschen R et al 2007 (6) & Yes & NA & Yes & Yes & 4 \\
Ducreux M et al $2010(7)$ & Yes & NA & Yes & Yes & 4 \\
Van Cutsem E et al $2009(8)$ & Yes & NA & Yes & Yes & 4 \\
Diaz-Rubio E et al $2007(9)$ & Yes & NA & Yes & Yes & 4 \\
Comella P et al $2009(10)$ & Yes & NA & Yes & Yes & 4 \\
Cassidy J et al 2008 (11) & Yes & NA & Yes & Yes & 4 \\
\hline
\end{tabular}

NA, not available; ITT, intention-to-treat analysis; lose, lost to follow-up. Total Jadad scores 1-5. 1-2: low quality studies, 3-5: high quality studies.

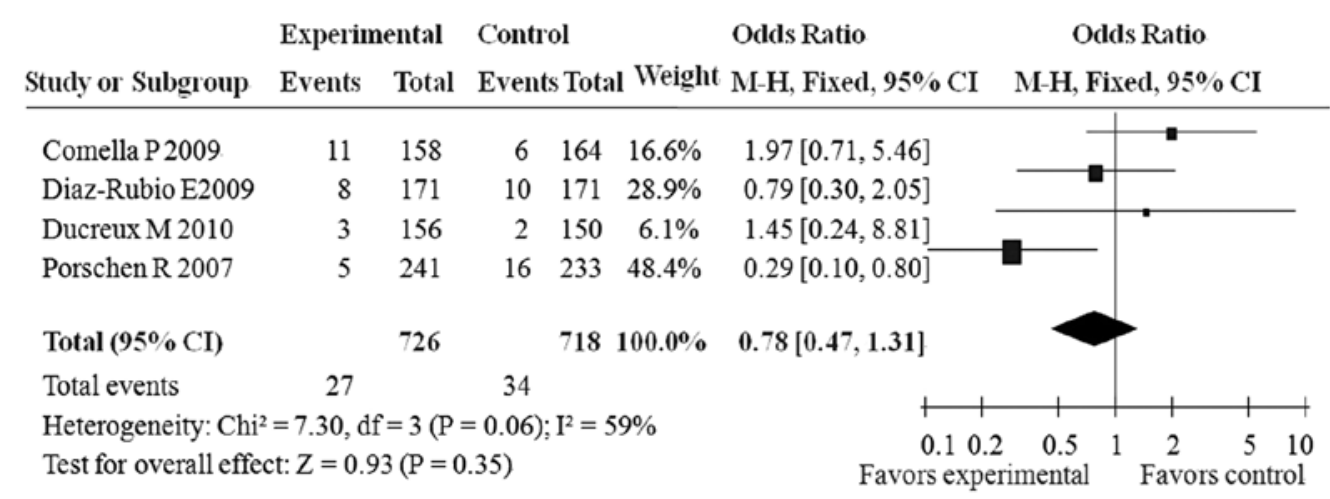

Figure 1. Meta-analysis of the complete response rate of capecitabine plus oxaliplatin compared with 5-fluorouracil plus oxaliplatin for metastatic colorectal cancer.

\begin{tabular}{|c|c|c|c|c|c|c|}
\hline Study or Subgroup & Events & Total & Event & s TotaWeightM & $-\mathrm{H}$, Fixed, $95 \%$ CI & M-H, Fixed, $95 \%$ CI \\
\hline Comella P 2009. & 42 & 158 & 48 & $164 \quad 18.6 \%$ & $0.88[0.54,1.42]$ & - \\
\hline Diaz-Rubio E2009 & 56 & 171 & 68 & $171 \quad 24.5 \%$ & $0.74[0.47,1.15]$ & \\
\hline Ducreux M 2010 & 58 & 156 & 68 & $150 \quad 23.4 \%$ & $0.71[0.45,1.13]$ & \\
\hline Porschen R 2007 & 110 & 241 & 113 & $233 \quad 33.5 \%$ & $0.89[0.62,1.28]$ & \\
\hline Total $(95 \%$ CI $)$ & & 726 & & $718 \quad 100.0 \%$ & $0.81[0.65,1.00]$ & \\
\hline Total events & 266 & & 297 & & & \\
\hline $\begin{array}{l}\text { Heterogeneity: } \mathrm{Chi}^{2} \\
\text { Test for overall effe }\end{array}$ & $\begin{array}{l}2=0.84, \mathrm{~d} \\
\mathrm{ct}: Z=1\end{array}$ & $\begin{array}{l}=3(\mathrm{P}= \\
4(\mathrm{P}=0 .\end{array}$ & $\begin{array}{l}=0.84) \\
.05)\end{array}$ & $\mathrm{I}^{2}=0 \%$ & Favor: & 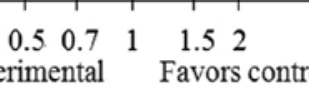 \\
\hline
\end{tabular}

Figure 2. Meta-analysis of the partial response rate of capecitabine plus oxaliplatin compared with 5-fluorouracil plus oxaliplatin for metastatic colorectal cancer.

The heterogeneity test appeared to show no statistically significant difference $\left(\mathrm{p}=0.05, \mathrm{I}^{2}=58 \%\right)$; thus we confirmed that the difference between the capecitabine plus oxaliplatin group and the 5-FU plus oxaliplatin group was statistically significant by using the fixed effect model (OR=2.71, 95\% CI 2.04-3.61, p<0.00001; Fig. 4), which revealed that the incidence of hand-foot syndrome in the capecitabine plus oxaliplatin group was markedly higher than that the 5-FU plus oxaliplatin group. Conversely, stomatitis and neutropenia were more commonly observed in the 5-FU plus oxaliplatin group. The third and fourth trials, respectively, reported stomatitis and neutropenia. Significant differences were not found $\left(\mathrm{p}=0.09, \mathrm{I}^{2}=59 \%\right)$ in the adverse reaction of stomatitis by using a fixed effects model; the resulting difference between the two regimens was statistically significant $(\mathrm{OR}=0.51,95 \% \mathrm{CI}$ 0.38-0.70, p<0.0001; Fig. 5). Regarding neutropenia, we used a random effects model $\left(\mathrm{p}<0.00001, \mathrm{I}^{2}=89 \%\right)$ and received a similar result to stomatitis $(\mathrm{OR}=0.31,95 \%$ CI $0.16-0.60$, 
Table III. Analytic results of median OS and PFS.

\begin{tabular}{|c|c|c|c|c|c|c|}
\hline \multirow[t]{2}{*}{ Study (year, reference) } & \multicolumn{3}{|c|}{ PFS (month) } & \multicolumn{3}{|c|}{ Median OS (month) } \\
\hline & Experimental group & Control group & P-value & Experimental group & Control group & P-value \\
\hline Rothenberg ML et al 2008 (5) & 4.7 & 4.8 & - & 11.9 & 12.6 & - \\
\hline Porschen R et al 2007 (6) & 7.1 & 8.0 & 0.117 & 16.8 & 18.8 & 0.26 \\
\hline Ducreux M et al 2010 (7) & 8.8 & 9.3 & - & 19.9 & 20.5 & - \\
\hline Van Cutsem E et al 2009 (8) & 10.8 & 11.3 & - & 23.0 & 25.9 & - \\
\hline Diaz-Rubio E et al 2007 (9) & 8.9 & 9.5 & 0.153 & 18.1 & 20.8 & 0.145 \\
\hline Comella P et al 2009 (10) & 6.6 & 6.5 & 0.699 & 16.0 & 17.1 & 0.883 \\
\hline Cassidy J et al 2008 (11) & 8.4 & 8.5 & - & 19.8 & 19.6 & - \\
\hline
\end{tabular}

PFS, progression-free survival; OS, overall survival.

Experimental Control

Odds Ratio

Study or Subgroup Events TotalEvents Total Weight M-H, Fixed, 95\% CI M-H, Fixed, 95\% CI

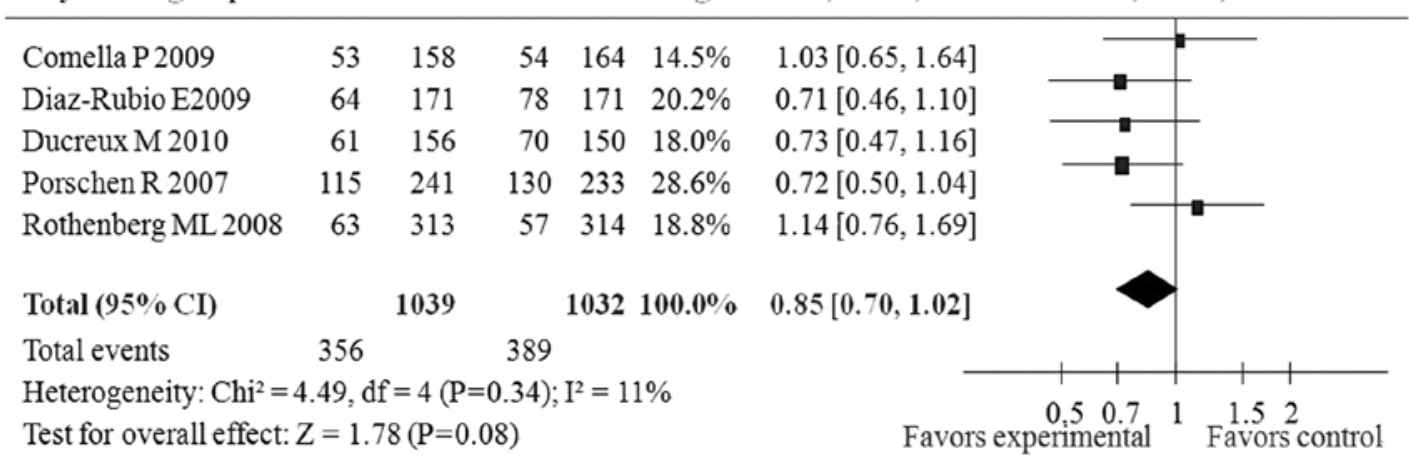

Figure 3. Meta-analysis of the overall response rate of capecitabine plus oxaliplatin compared with 5-fluorouracil plus oxaliplatin for metastatic colorectal cancer.

Control Odds Ratio Odds Ratio

Study or Subgroup Events TotalEvents Total Weight $\quad$ M-H, Fixed, 95\% CI $\quad$ M-H, Fixed, 95\% CI

\begin{tabular}{|c|c|c|c|c|c|c|}
\hline Comella P2009 & 23 & 158 & 17 & $164 \quad 23.4 \%$ & $1.47[0.75,2.88]$ & \\
\hline Diaz-Rubio E2009 & 28 & 171 & 11 & $171 \quad 15.1 \%$ & $2.85[1.37,5.93]$ & \\
\hline Ducreux M 2010 & 32 & 156 & 19 & $150 \quad 25.3 \%$ & $1.78[0.96,3.30]$ & \\
\hline Porschen R 2007 & 24 & 241 & 9 & $233 \quad 13.5 \%$ & $2.75[1.25,6.06]$ & \\
\hline Rothenberg ML 2008 & 72 & 313 & 18 & $314 \quad 22.7 \%$ & $4.91[2.85,8.46]$ & \\
\hline Total $(95 \% \mathrm{CI})$ & & 1039 & & $1032100.0 \%$ & $2.71[2.04,3.61]$ & \\
\hline Total events & 179 & & 74 & & & \\
\hline
\end{tabular}

Figure 4. Meta-analysis of the incidence of hand-foot syndrome using capecitabine plus oxaliplatin compared with 5-fluorouracil plus oxaliplatin for metastatic colorectal cancer.

p=0.0005; Fig. 6). However, certain common toxic effects, for example, nausea, vomiting, diarrhea, fever, thrombocytopenia and peripheral neuropathy, occurred to a similar extent in the two regimens ( $>00.05)$. Remaining data on adverse events are shown in Table IV.

\section{Discussion}

The drug 5-FU has been the main chemotherapy for colorectal carcinoma in clinical use as it was the only available drug. Recently, improved regimens combining 5-FU with oxaliplatin 
Table IV. Meta-analysis of partial adverse events.

\begin{tabular}{|c|c|c|c|c|c|c|c|c|c|}
\hline \multirow[t]{2}{*}{ Adverse reaction } & \multirow{2}{*}{$\begin{array}{r}\mathrm{RCT} \\
(\mathrm{n})\end{array}$} & \multirow{2}{*}{$\begin{array}{l}\text { Experimental } \\
\text { group }(\mathrm{n} / \mathrm{N})\end{array}$} & \multirow{2}{*}{$\begin{array}{l}\text { Control group } \\
(\mathrm{n} / \mathrm{N})\end{array}$} & \multicolumn{2}{|c|}{ Heterogeneity test } & \multirow[t]{2}{*}{ Effect model } & \multirow[t]{2}{*}{ OR } & \multirow[t]{2}{*}{$95 \% \mathrm{CI}$} & \multirow[t]{2}{*}{ P-value } \\
\hline & & & & P-value & $\mathrm{I}^{2} \%$ & & & & \\
\hline Nausea & 3 & $357 / 640$ & $360 / 635$ & 0.02 & 73 & Random & 0.89 & $0.57-1.39$ & 0.61 \\
\hline Diarrhea & 4 & $417 / 798$ & $420 / 799$ & 0.0004 & 84 & Random & 0.91 & $0.55-1.51$ & 0.71 \\
\hline Fever & 3 & $124 / 640$ & $130 / 635$ & 0.99 & 0 & Fixed & 0.94 & $0.71-1.23$ & 0.64 \\
\hline Thrombocytopenia & 4 & 206/798 & $250 / 799$ & 0.02 & 71 & Random & 0.73 & $0.48-1.13$ & 0.16 \\
\hline Peripheral neuropathy & 3 & $177 / 712$ & $182 / 711$ & 0.38 & 0 & Fixed & 0.97 & $0.75-1.24$ & 0.79 \\
\hline
\end{tabular}

Experimental Control

Study or Subgroup $\quad$ Events $\quad$ TotaEvents TotaWeight $\quad$ M-H, Fixed,95\% CI $\quad$ M-H, Fixed, 95\% CI

\begin{tabular}{|c|c|c|c|c|c|c|}
\hline Comella P 2009 & 24 & 158 & 29 & $16420.4 \%$ & $0.83[0.46,1.51]$ & \\
\hline Ducreux M 2010 & 11 & 156 & 15 & $150 \quad 12.0 \%$ & $0.68[0.30,1.54]$ & \\
\hline Rothenberg ML 2008 & 44 & 313 & 93 & $314 \quad 67.5 \%$ & $0.39[0.26,0.58]$ & \\
\hline Total $(95 \% \mathrm{CI})$ & & 627 & & $628100.0 \%$ & $0.51[0.38,0.70]$ & \\
\hline Total events & 79 & & 137 & & & \\
\hline $\begin{array}{l}\text { Heterogeneity: } \mathrm{Chi}^{2}= \\
\text { Test for overall effect: }\end{array}$ & $\begin{array}{l}91, \mathrm{df} \\
=4.2\end{array}$ & $\begin{array}{l}=2(\mathrm{P} \\
(\mathrm{P}<0\end{array}$ & $\begin{array}{l}.09) ; \\
01)\end{array}$ & $=59 \%$ & $\begin{array}{ccc}0.01 & 0.1 & 1 \\
\text { Favors experimental } & \end{array}$ & $\begin{array}{cc}10 & 100 \\
\text { Favors control }\end{array}$ \\
\hline
\end{tabular}

Figure 5. Meta-analysis of the incidence of stomatitis in capecitabine plus oxaliplatin compared with 5-fluorouracil plus oxaliplatin for metastatic colorectal cancer.

Control

Study or Subgroup Events Total Events TotaWeight M-H, Random, $95 \%$ CI M-H, Random, $95 \%$ CI

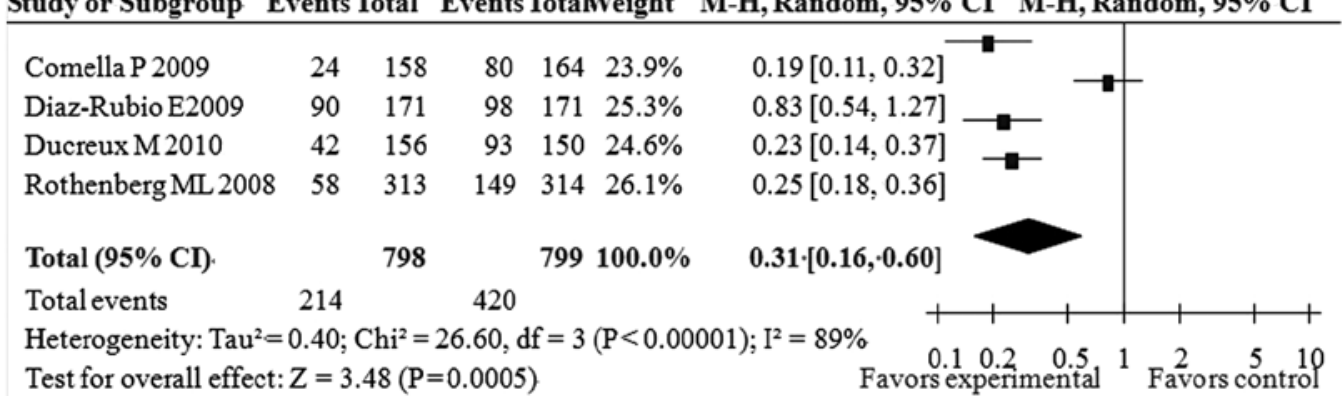

Figure 6. Meta-analysis of the incidence of neutropenia in capecitabine plus oxaliplatin compared with 5-fluorouracil plus oxaliplatin for metastatic colorectal cancer.

have been applied, which may be considered as more efficacious. Oxaliplatin is a third-generation cisplatin derivative with anti-cancer activity. The mechanism of action, platinum atom and DNA, form the platinum-DNA bulky adducts, which are capable of restraining DNA synthesis and repair, and activating the cell signaling pathway through intrastrand, interstrand and protein cross-linking with DNA. Oxaliplatin was found to be highly active as it was higher and firmer when combined with DNA than other platinum drugs. De Gramont et al demonstrated significantly higher activity of a combination of oxaliplatin plus 5-FU/LV compared with 5-FU/LV as first-line therapy in advanced CRC in a phase III trial (12). The combined regimen of oxaliplatin plus 5-FU/LV as firstline chemotherapy for advanced CRC also demonstrated good tolerance and more effective power of $34-67 \%$ (13).
Capecitabine (Xeloda; Hoffmann-La Roche Inc.), an oral prodrug of fluoropyrimidine, is absorbed from the gastrointestinal tract in an inactive form and generates fluorouracil by way of a three-step enzymatic cascade. The final stage of doxifluridine converted to fluorouracil is catalyzed by the enzyme thymidine phosphorylase (TP), which is present in tumors at a higher concentration than in normal tissue (14). Cassidy et al confirmed that oxaliplatin upregulates the expression of TP in a CXF280 xenograft model of human colon tumor tissue to increase the collaborative anti-cancer activity with capecitabine (15). Early phase II trials considering the XELOX (oxaliplatin $130 \mathrm{mg} / \mathrm{m}^{2}$ on day 1 every 3 weeks) and CAPOX (oxaliplatin $70 \mathrm{mg} / \mathrm{m}^{2}$ on days 1 and 8 every 3 weeks) regimens showed an ORR of $37-49 \%$, and the endpoints of median TTP and median OS were 5.9 to 8.2 months and 15.8 to 20 months, 
respectively (16-18). Recently, more attention has been paid to combination chemotherapy of CRC with the regimen of capecitabine and oxaliplatin for safe, reliable and convenient medication. In certain randomized studies it was concluded that XELOX is non-inferior in terms of efficacy to the FOLFOX regimen in the first-line treatment of MCRC $(5-7,9,11)$. In addition, as a substitute of 5-FU, capecitabine did not require central venous catheterization, preventing infection, thromboembolism and certain other risks in elderly patients. Results of the study by Comella et al on 76 elderly patients with MCRC (mean age, 75 years) receiving the XELOX regimen, with an ORR of $41 \%$ (95\% CI 30-53\%), including 2 CR and 29 PR, showed the median PFS to be 8.5 months (95\% CI 6.7-10.3 months), and the median OS to be 14.4 months (95\% CI 11.9-16.9 months). Regarding adverse reactions during treatment, $5 \%$ of patients had Grade $\geq 3$ hematologic toxicity, $8 \%$ of patients had Grade 3 peripheral neuropathy and $13 \%$ of patients had severe hand-foot syndrome (19). Similar results were obtained in another phase II trial that included a total of 50 patients aged $>70$ years with MCRC, using the XELOX regimen as first-line therapy. The authors observed that the ORR was 36\% (95\% CI 28-49\%), with $3 \mathrm{CR}$ and $15 \mathrm{PR}$. The median OS was 13.2 months (95\% CI 7.6-16.9 months). There were 14 (28\%) patients who exhibited Grade $\geq 3$ adverse reactions including 11 (22\%) with diarrhea, $8(16 \%)$ with asthenia, 7 (14\%) with nausea/vomiting, $3(6 \%)$ with neutropenia, $3(6 \%)$ with thrombocytopenia and 2 (4\%) with hand-foot syndrome (20). Based on the data from these trials, capecitabine and oxaliplatin may be regarded as an appropriate treatment selection for elderly patients with MCRC. This combined therapy may be well tolerated and have reliable clinical efficacy.

Our study analyzed published trials comparing capecitabine plus oxaliplatin with 5-FU plus oxaliplatin in patients with MCRC in the last decade. Analyses were according to the intention-to-treat principle. The results revealed that $\mathrm{CR}, \mathrm{PR}$ and ORR had similar curative effects between the capecitabine plus oxaliplatin group and the 5-FU plus oxaliplatin group, and the median OS and PFS had no statistically significant differences. Regarding safety, hand-foot syndrome was more frequently observed in the capecitabine plus oxaliplatin group, while stomatitis and neutropenia were reversed. Other toxic effects had no statistically significant differences between the two groups. Our findings have shown that capecitabine plus oxaliplatin had similar curative effects as 5-FU plus oxaliplatin, but was safer in patients with MCRC.

Since capecitabine is a prodrug of fluoropyrimidine, it is capable of reducing the systemic exposure of the active form of 5-FU to normal tissue. In 2001, a large Phase III randomized trial compared capecitabine with intravenous 5-FU in patients with MCRC (21). The toxicity of capecitabine mainly included hand-foot syndrome $(\mathrm{p}<0.0001)$, but resulted in lower incidences of Grade 3/4 stomatitis and neutropenia compared to 5-FU ( $\mathrm{p}<0.00001)$. The results are consistent with our study. Results of another study (22) found that Grade 3 adverse events were more common in the capecitabine than those in the 5-FU/leucovorin group (38.1 vs. $34.1 \%$; $\mathrm{p}=0.16$ ), primarily due to Grade 3 hand-foot syndrome. However, Grade 4 adverse events were more frequent with 5-FU/leucovorin (3.0 vs. 5.1\%; $\mathrm{p}=0.078)$, which was mainly ascribed to neutropenia and diarrhea. The incidence of Grade 3 or 4 chemotherapy-related adverse events during the first treatment cycle was significantly higher in patients receiving 5-FU/leucovorin than capecitabine (22.6 vs. 9.1\%; p<0.001) (22). However, in our study, the incidence of diarrhea had no significant difference between the two regimens. More studies with high quality and large samples are required to update the investigation into adverse events.

Our report has a number of limitations. Firstly, we did not obtain information from each individual patient for each trial. Furthermore, PFS and median OS belong to the skewed distribution that cannot be assessed by meta-analysis. Secondly, there is a potential bias in the studies included, as the dose and specification of chemotherapy drugs were not uniform. Thirdly, unpublished trials were not included. Finally, the result of the comparison between certain adverse effects is not stable and reliable enough due to the small number of inclusive studies. We believe that more results with improved methodological quality should be provided to update this study. Should findings of the present study be utilized as a reference, it would be necessary to make a reasonable adjustment for other research results, clinical experience and the individual patient characteristics in clinical practice.

\section{References}

1. Jemal A, Siegel R, Ward E, et al: Cancer statistics. CA Cancer J Clin 57: 43-66, 2009.

2. Goldberg RM, Rothenberg ML, Van Cutsem E, et al: The continuum of care: a paradigm for the management of metastatic colorectal cancer. Oncologist 12: 38-50, 2007.

3. Hoff PM, Ansari R, Batist G, et al: Comparison of oral capecitabine versus intravenous fluorouracil plus leucovorin as first-line treatment in 605 patients with metastatic colorectal cancer: results of a randomized phase III study. Clin Oncol 19: 2282-2292, 2001.

4. Jadad AR, Moore RA, Carroll D, et al: Assessing the quality of reports of randomized clinical trials: Is blinding necessary? Control Clin Trials 17: 1-12, 1996.

5. Rothenberg ML, Cox JV, Butts C, et al: Capecitabine plus oxaliplatin (XELOX) versus 5-fluorouracil/folinic acid plus oxaliplatin (FOLFOX-4) as second-line therapy in metastatic colorectal cancer: a randomized phase III noninferiority study. Ann Oncol 19: 1720-1726, 2008.

6. Porschen R, Arkenau HT, Kubicka S, et al: Capecitabine plus oxaliplatin compared with fluorouracil and leucovorin plus oxaliplatin: a randomized comparison in metastatic colorectal cancer-a final report of the AIO Colorectal Study Group. J Clin Oncol 25: 4217-4223, 2007.

7. Ducreux M, Bennouna J, Hebbar M, et al: Capecitabine plus oxaliplatin (XELOX) versus 5-fluorouracil/leucovorin plus oxaliplatin (FOLFOX-6) as first-line treatment for metastatic colorectal cancer. Int J Cancer 128: 682-690, 2011.

8. Van Cutsem E, Rivera F and Berry S: Safety and efficacy of first-line bevacizumab with FOLFOX, XELOX, FOLFIRI and fluoropyrimidines in metastatic colorectal cancer: the BEAT study. Ann Oncol 20: 1842-1847, 2009.

9. Diaz-Rubio E, Tabernero J, Gomez-Espana A, et al: Phase III study of capecitabine plus oxaliplatin versus continuous infusion fluorouracil plus oxaliplatin as first-line therapy in metastatic colorectal cancer: final report of the Spanish Cooperative Group for the Treatment of Digestive Tumors trial. J Clin Oncol 25: 4224-4230, 2007.

10. Comella P, Massidda B, Filippelli G, et al: Randomised trial comparing biweekly oxaliplatin plus oral capecitabine versus oxaliplatin plus i.v. bolus fluorouracil/ leucovorin in metastatic colorectal cancer patients: results of the Southern Italy Cooperative Oncology study 0401. J Cancer Res Clin Oncol 135: 217-226, 2009.

11. Cassidy J, Clarke S, Diaz-Rubio E, et al: A randomized phase III study of capecitabine plus oxaliplatin (XELOX) versus fluorouracil/folinic acid plus oxaliplatin (FOLFOX-4) as first-line therapy for metastatic colorectal cancer. J Clin Oncol 26: 2006-2012, 2008. 
12. de Gramont A, Figer A, Seymour M, et al: Leucovorin and fluorouracil with or without oxaliplatin as first-line treatment in advanced colorectal cancer. J Clin Oncol 18: 2938-2947, 2000.

13. Kouroussis C, Souglakos J, Kakolyris S, et al: Oxaliplatin in combination with infusional 5-fluorouracil and leucovorin every 2 weeks as first-line treatment in patients with advanced colorectal cancer: a phase II study. Oncology 61: 36-41, 2001.

14. Miwa M, Ura M, Nishida M, et al: Design of a novel oral fluoropyrimidine carbamate, capecitabine, which generates 5 -fluorouracil selectively in tumours by enzymes concentrated in human liver and cancer tissue. Eur J Cancer 34: 1274-1281, 1998.

15. Cassidy J and Tabernero J: XELOX (capecitabine plus oxaliplatin): active first-line therapy for patients with metastatic colorectal cancer. J Clin Oncol 22: 2084-2091, 2004.

16. Borner MM, Dietrich D, Stupp R, et al: Phase II study of capecitabine plus oxaliplatin compared with fluorouracil and leucovorin plus oxaliplatin in first and second line treatment of advanced or metastic colorectal cancer. J Clin Oncol 20 1759-1766, 2002

17. Zeuli M, Nardoni C, Pino MS, et al: Phase II study of capecitabine and oxaliplatin as first-line treatment in advanced colorectal cancer. Ann Oncol 14: 1378-1382, 2003.
18. Shields AF, Zaupski MM, Marshall JL, et al: Treatment of advanced colorectal carcinoma with oxaliplatin and capecitabine: a phase II trial. Cancer 100: 531-537, 2004.

19. Comella P, Natale D, Farris A, et al: Capecitabine plus oxaliplatin for the first-line treatment of elderly patients with metastatic colorectal carcinoma final results of the southern Italy Cooperative Oncology Group Trial 0108. Cancer 104: 282-289, 2005.

20. Feliu J, Salud A, Escudero P, et al: XELOX (Capecitabine plus oxaliplatin) as first-line treatment for elderly patients over 70 years of age with advanced colorectal cancer. Br J Cancer 94: 969-975, 2006.

21. Van Cutsem E, Twelves C, Cassidy J, et al: Oral capecitabine compared with intravenous fluorouracil plus leucovorin in patients with metastatic colorectal cancer: results of a large phase III study. J Clin Oncol 19: 4097-4106, 2001.

22. Cassidy J, Twelves C, Van Cutsem E, et al: First-line oral capecitabine therapy in metastatic colorectal cancer: a favorable safety profile compared with intravenous 5-fluorouracil/ leucovorin. Ann Oncol 13: 566-575, 2002. 